\title{
The Impairment of Trans Fatty Acids on Learning, Memory and Brain Amino Acid Neurotransmitters in Mice
}

\author{
Tiantian ZHAng, Ping LiU*, Yu Sun, Na ReN and Shuang NIE \\ Department of Hygiene Detection, College of Public Health, Shandong University, \\ Jinan, Shandong, 250012, China
}

(Received June 1, 2017)

\begin{abstract}
Summary The aim of the study was to demonstrate the impairment of trans fatty acids (TFAs) in neurological disorders in mice. Forty-eight male Kunming mice were randomly divided into four groups with twelve in each group, namely a control group (corn oil group), and TFA groups with low, middle and high dosages. The tested chemicals were given by gavage, once a day, for $12 \mathrm{wk}$ in total, with the volume of the intragastric liquid as $0.1 \mathrm{~mL} / 10 \mathrm{~g}$ of body weight. The mice in the control group were given corn oil only. The mice in the TFA groups were given TFA solution (doses were 25,50 and $100 \mathrm{mg} / \mathrm{kg}$ of body weight, respectively). The Morris circle water maze was used to test learning and memory of mice. The activity of acetylcholinesterase (AChE), nitric oxide synthase (NOS) and the level of brain amino acid neurotransmitters were tested. In the Morris water maze task, and compared to the control group, TFAs showed no obvious effect on learning or memory. TFA intake led to a significant decrease of AChE in all TFA groups, and the increase in levels of NOS in the high-dose group. Meanwhile, intake of TFAs increased the levels of Asp, Glu, Gly, and Tau in all TFA groups. The results suggest that long-term intake of TFAs probably impairs learning, memory and brain neurotransmitters in mice.
\end{abstract}

Key Words trans fatty acids, learning and memory, AChE, NOS, neurotransmitters

Trans fatty acids (TFAs) are the general name of unsaturated fats with at least one double bond in the trans configuration (1). TFAs in food are usually derived from three main sources, namely catalytic hydrogenation of oils and fats, biotransformation by bacteria and heat treatments. TFAs may be used in a variety of manufactured foods, especially partially hydrogenated oils and their products. Partially hydrogenated oils contain up to $30-50 \%$ TFAs, which may account for the presence of TFAs in certain food categories. Previous studies suggest that intake of TFAs is only a nutritional problem, but a growing number of data from both toxicological studies and epidemiology assessment show that TFAs have many adverse effects on human health. The association between cardiovascular disease and TFA intake has been extensively described, especially with coronary heart disease (2). In addition, some studies suggest that TFA intake is associated with the development of diabetes (3), delayed infant and fetal development (4), Alzheimer's disease (AD) (5), and even cancers (6). However, these associations have not been extensively studied.

Early in the 1990s, it was reported that people with bad dietary habits in adolescence, were much likelier to get $\mathrm{AD}$. The intake of TFAs increases the accumulation of insoluble amyloid- $\beta(\mathrm{A} \beta)(7)$, affects the cell oxidative stress level (8), and leads to hypothalamic inflammation (9). It can inhibit the activities of $\mathrm{Na}^{+} \mathrm{K}^{+}$-ATPase (10) and decreases the contents of DHA (11).

\footnotetext{
*To whom correspondence should be addressed.

E-mail: liupingp@sdu.edu.cn
}

In our study, we hypothesized that long-term intake of TFAs may cause impairment to the brain. Morris water maze tasks were used to evaluate the effects of TFAs on learning and memory in mice. The changes in activities of nitric oxide synthase (NOS) and acetylcholinesterase (AChE), and the contents of brain amino acid neurotransmitters (Asp, Glu, Gly, GABA and Tau) were determined to evaluate the effects of TFAs on neurological disorders in mice.

\section{MATERIALS AND METHODS}

Materials. Trans fatty acid (mass fraction: 40\%) was purchased from Yuan Cheng Group (Wuhan, China). The test kits for total protein (TP), NOS and AChE were all purchased from Nanjing Jiancheng Bioengineering Institute, China. Acetonitrile and methanol (HPLCgrade) were procured from Cinc High Parity Solvents Co., Ltd. (Shanghai, China). Neurotransmitter standard substances were obtained from the National Institute for the Control of Pharmaceutical and Biological Products. 2,4-Dinitrofluorobenzene (DNFB) was obtained from Baoman Biological Technology Co., Ltd. (Shanghai, China). All other reagents were of analytical-reagent grade.

Treatment of animals. Healthy male Kunming mice $(n=48)$ were purchased from Central Animal House, Shandong University (Jinan, China). Mice were acclimated to the laboratory conditions for $1 \mathrm{wk}$, with the controlled conditions $\left(20-24^{\circ} \mathrm{C}, 50-60 \%\right.$ humidity) of a $12 \mathrm{~h}$ light/dark cycle. Food and water were freely supplied. The protocol was approved by the Institutional 
Animal Ethics Committee and all animal studies were compliant with Animal Care and Use Guideline in China (approval number: 20120601).

Mice were randomly assigned into four groups (12 mice in each), namely, a control group, and TFA groups with low (L-TFA), middle (M-TFA) and high (H-TFA) dosages. In the control group, intragastric administration of corn oil was carried out. And intragastric administration of different dosages of TFA with 25, 50 and $100 \mathrm{mg} / \mathrm{kg}$ of body weight was also used in the TFA groups with low, middle and high dosages. All solutions above were administrated via oral gavage every day for $12 \mathrm{wk}$.

Morris water maze experiment. Mice in all groups were studied for their spatial memory and learning abilities through the Morris water maze tests.

The Morris water maze experiment was conducted in two phases, the hidden platform test and the probe trial. In the hidden platform test, each mouse was placed in one of the four quadrants. For each trial, mice were required to search for the hidden platform for a maximum of $120 \mathrm{~s}$. If a mouse didn't find the platform within $120 \mathrm{~s}$, it was guided to the platform, and stayed for $30 \mathrm{~s}$. We recorded the time for $120 \mathrm{~s}$. Each mouse performed in the four trials for four consecutive days. We recorded the daily escape latency and the swimming distance. The fifth day was the probe trial. We removed the hidden platform, give the mice $60 \mathrm{~s}$ to swim in the pool to measure the spatial bias, and recorded the swimming time in the quadrant of the platform and the number of crossings over the previous platform location.

Determination of the activity of nitric oxide synthase (NOS) and acetylcholinesterase (AChE) in the brain of mice. We accurately weighed an appropriate amount of brain and made up a $10 \%$ brain homogenate with saline.

The content of total protein, and the activities of NOS and AChE were measured immediately, following the kit instructions. The content of total protein was determined using Coomassie Brilliant Blue at $595 \mathrm{~nm}$. The activity of NOS was measured by the content of NO at $530 \mathrm{~nm}$. The activity of AChE was assayed at $412 \mathrm{~nm}$.

Determination of the contents of amino acid neurotransmitters in the brain of mice. The contents of aspartic acid (Asp), glutamate (Glu), glycine (Gly), $\gamma$-aminobutyric acid (GABA) and taurine (Tau) were tested by high performance liquid chromatography (HPLC).

The samples were accurately weighed and homogenized with acetonitrile/water $(1: 1, \mathrm{v} / \mathrm{v})$. The homogenate was centrifuged at $12,500 \times g$ for $10 \mathrm{~min}$ at $4^{\circ} \mathrm{C}$. Three hundred milliliters of the supernatant, $200 \mathrm{~mL}$ of $0.5 \mathrm{M} \mathrm{NaHCO}_{3}$ and $100 \mathrm{~mL}$ of $0.5 \%$ DNFB solution were mixed, then the derivatization reaction was carried out in a water bath $\left(65^{\circ} \mathrm{C}\right)$ for $55 \mathrm{~min}$ in the dark. After cooling, the solution was transferred and filtered through a $0.45 \mathrm{~mm}$ nylon filter into auto-sampler vials for injection.

Statistical analysis. Statistical analysis was carried out with SPSS, and results were expressed as mean \pm SD. Statistical analysis was determined by using one-way
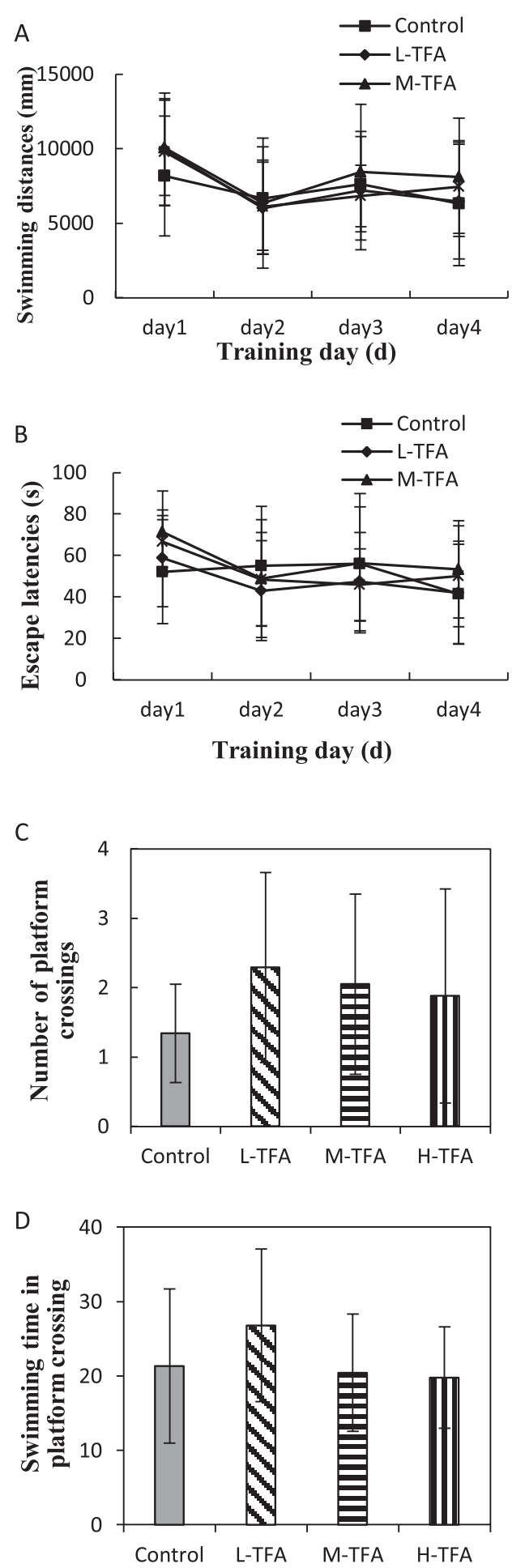

Fig. 1. On training days, the swimming distances (A) and the escape latencies (B) were recorded. The probe trials indicate the numbers of platform crossings (C) and the swimming time in the platform quadrant (D).

analysis of variance (ANOVA), and multiple comparisons between groups were made by using the StudentNewman-Keuls (SNK) test. Differences were considered significant if the $p$ value was $<0.05$.

\section{RESULTS}

Effects of TFA on learning and memory in mice

In the training stage, the escape latencies and the 


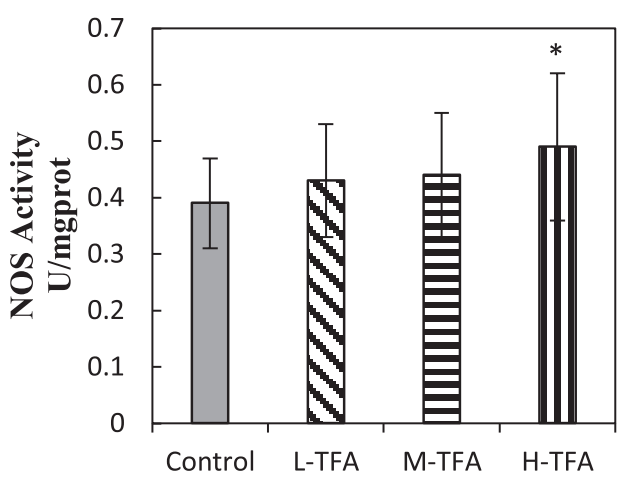

Fig. 2. Effects of TFA on the activity of NOS of mice (mean $\pm \mathrm{SD}, \mathrm{U} / \mathrm{mg}$ prot). * represents statistical significance $(p<0.05)$ as compared to the control group.

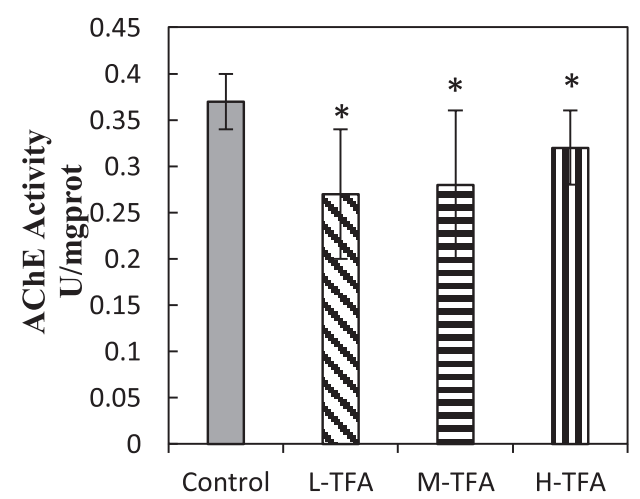

Fig. 3. Effects of TFA on the activity of AChE in mice (mean $\pm \mathrm{SD}, \mathrm{U} / \mathrm{mg}$ prot). * represents statistical significance $(p<0.05)$ as compared to the control group.

Table 1. Effects of TFA on brain amino acid neurotransmitter levels. The concentration of Asp, Glu, Gly, Tau and GABA is expressed as $\mu \mathrm{g} / \mathrm{g}$ tissue (mean $\pm \mathrm{SD})$.

\begin{tabular}{llllll}
\hline Group & \multicolumn{1}{c}{ Asp } & \multicolumn{1}{c}{ Glu } & \multicolumn{1}{c}{ Gly } & Tau & GABA \\
\hline Control & $10.66 \pm 1.59$ & $22.22 \pm 2.73$ & $36.23 \pm 1.69$ & $16.58 \pm 3.51$ & $6.10 \pm 0.39$ \\
L-TFA & $22.97 \pm 3.53^{*}$ & $42.94 \pm 9.53^{*}$ & $66.94 \pm 7.00^{*}$ & $22.78 \pm 2.01^{*}$ & $7.52 \pm 0.49$ \\
M-TFA & $20.71 \pm 5.65^{*}$ & $36.25 \pm 8.85^{*}$ & $65.97 \pm 11.21^{*}$ & $23.83 \pm 5.49^{*}$ & $7.11 \pm 1.61$ \\
H-TFA & $17.10 \pm 6.49$ & $28.96 \pm 9.76$ & $62.98 \pm 18.88^{*}$ & $18.69 \pm 4.12$ & $5.79 \pm 1.56$ \\
\hline
\end{tabular}

* represents statistical significance $(p<0.05)$ as compared to the control group.
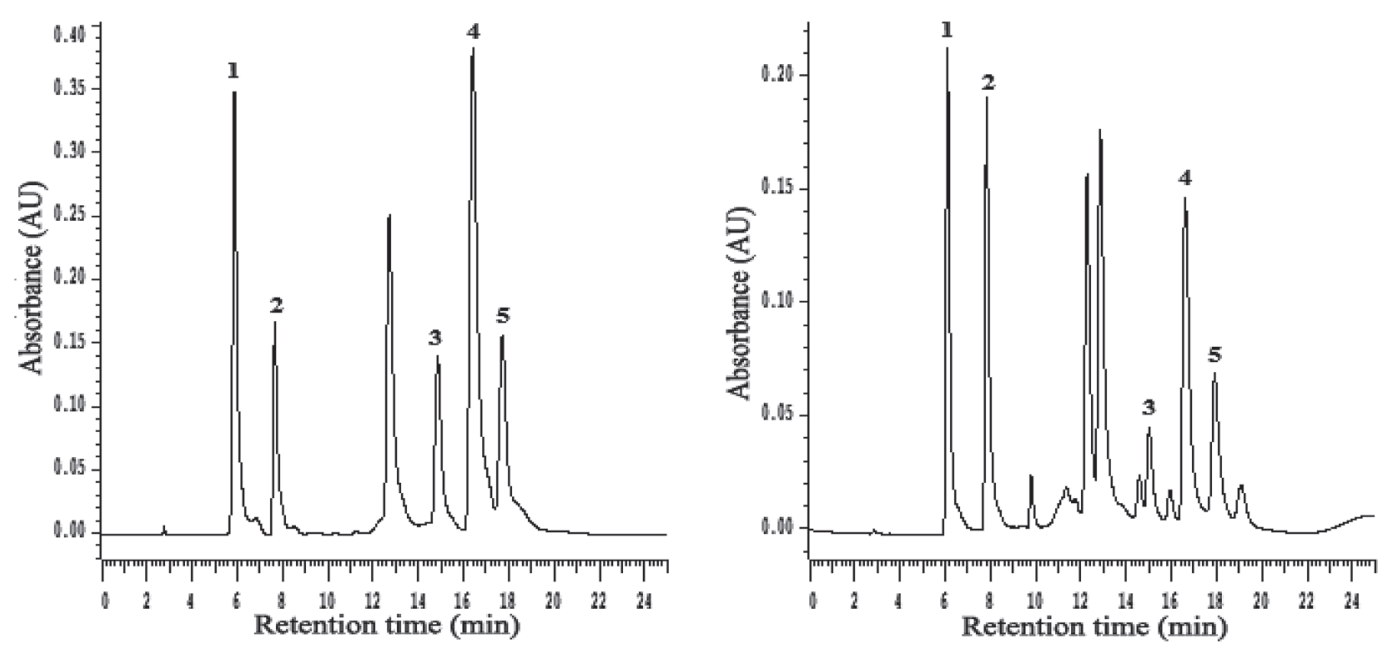

Fig. 4. Chromatograms of HPLC separation of amino acid standards (A) and samples (B); and peaks were identified as

(1) aspartic acid (Asp), (2) glutamic acid (Glu), (3) glycine (Gly), (4) taurine (Tau) and (5) $\gamma$-aminobutyric acid (GABA).

total swimming distances in searching for the hidden underwater platform decreased progressively with the increased training days. However, no significant differences were found when compared to those of the control group ( $p>0.05)$ (Fig. 1A, B).

In the testing stage, compared to the control group, the TFA groups showed no obvious differences in the swimming time in the quadrant of the platform or the numbers of crossings of the platform (Fig. 1C, D). Effects of TFA on the activity of NOS and AChE in mice

The activity of NOS and AChE in the brain of mice is shown in Fig. 2 and Fig. 3. Compared to the control group, NOS activity in the TFA groups showed an apparent increase, but only in the group treated with $100 \mathrm{mg}$ TFA $/ \mathrm{kg}$ did the difference reach the level of statistical significance $(p<0.05)$. Significant decreases in AChE activity were observed in all TFA groups $(p<0.05)$, as compared to the activity in the control group.

Effects of TFA on the contents of amino acid neurotransmitters in mice

As shown in Table 1, the contents of Asp, Glu, Gly, and Tau in all TFA groups showed increases compared to the control group. The L-TFA and M-TFA groups showed significant increases in their levels of Asp, Glu, Gly and 
Tau compared to the control group $(p<0.05)$. The level of GABA showed no significant differences among the four groups $(p>0.05)$.

The chromatograms of amino acid standard substances and samples are shown in Fig. 4.

\section{DISCUSSION}

Trans fatty acids (TFAs) are widely used in the food industry, because of their low cost and longer shelf life. Some epidemiological studies and animal experiments have reported significant inverse association between TFAs and neurological disorders. Grimm et al. demonstrated that TFA can increase amyloidogenic and decrease non-amyloidogenic procession of the amyloid precursor protein (APP), and increase the production of amyloid beta $(\mathrm{A} \beta)$ peptides, which are the characteristic neuropathological indicators of Alzheimer's disease (AD) (7). In the present study, we have investigated whether oral intake of TFAs can induce behavioral and biochemical impairment in mice brains.

In several studies, a significant inverse relationship between TFAs and memory or cognition was reported. Golomb and Bui (12) reported that greater TFA was significantly associated with worse word recall in younger adults. However, in the present Morris water maze task, TFAs didn't have an obvious effects on the ability to learn or remember in mice. TFAs are associated with the activation of systemic inflammatory responses, and have an effect on endothelial function (13). On the basis of the mechanism, long-term intake of TFAs can lead to the developmental disorder of nerve endothelial cells, decrease the nerve excitation and the activity of freedom movement. This is not consistent with the present study. The possible reason is that the exposure time was too short, or the selected dose was too low, and failed to achieve the threshold of animal behavior changes. So a long-term and high-dose experimental study is also very necessary.

The activity of NOS and AChE can also reflect learning ability and memory in mice. Nitric oxide (NO) can maintain the long-term potentiation (LTP) to affect the learning and memory (14). It is currently thought that LTP is the main mechanism of synaptic plasticity and learning-memory. Therefore, NO plays an important role in neurotransmission and neuromodulation. NOS is the key enzyme and rate-limiting enzyme in the process of NO synthesis. In this experiment, the NOS activity showed an apparent increase in TFA groups compared with the control group. It is indicated that intake of TFAs may increase the level of NO and NOS. The disorder of NO will destroy the balance of free radicals, lead to the metabolism disorder in active oxygen and free radicals inside the body, and eventually result in the lesion of the mouse brain.

Acetylcholine (ACh), the principal neurotransmitter in the nervous system, is closely related with the formation of memory trace and the enhancement of long-term memory. The activity of AChE can indirectly reflect the ACh level. In the present study, compared to that of the control group, the level of AChE in the TFA groups was obviously decreased $(p<0.05)$. It is indicated that TFAs are potential inhibitors of AChE. It can result in the mass accumulation of ACh in the synaptic cleft, which acts on the cholinergic receptor and causes the disorder of the cholinergic system.

The amino acid neurotransmitters, widely distributed in the central nervous system (CNS), are amino acids which play an important role in transmitting a nerve message across a synapse. Glu and Asp are excitatory amino acids (EAAs), which are released by the presynaptic membrane. They selectively bind to G-proteinmediated Glu-receptors and NMDA-receptors, open the calcium channels, increase the $\mathrm{Ca}^{2+}$ influx, and consequently participate in LTP generation and maintenance $(15,16)$. However, Glu at high concentrations may produce an excitatory toxicity. It can lead to the overactivation of NMDA receptors, causing an increase in $\mathrm{Ca}^{2+}$ influx. In our present study, the concentration of Glu and Asp were increased in all TFA groups. It is indicated that intake of TFAs can cause a large increase in the EAAs in the brain, trigger Glu-mediated neurotoxicity, and induce an over-excited reaction of the brain. But in the high-dose TFA group, Glu and Asp contents were decreased, which indicates that high-dose intake of TFAs can induce neurotransmitter imbalance. GABA, Gly and Tau are the main inhibitory amino acids (IAAs); they negatively regulate the ability of learning and memory, and play a key role in modulating the excitatoryinhibitory balance in the brain (17). The increase of GABA, Gly and Tau release will inhibit the Glu-mediated $\mathrm{Ca}^{2+}$ influx, and resist the neurotoxicity caused by Glu release. This may be a protective mechanism. We found that TFA intake significantly increased the inhibitory neurotransmitter contents. This suggests that IAAs may play a neuroprotective role against the excessive release of EAAs. Similarly, it is also manifested that excessive intake of TFAs can cause the disorder.

Taken together, we can determine that the excitatory and inhibitory neurotransmitters maintain a balance in the CNS. Similarly, it's concluded that TFAs can influence the levels of both excitatory and inhibitory neurotransmitters in the mouse brain.

The present study validates the fact that excessive and long-term intake of TFAs probably causes an increase of NOS positive expression and disorder of AChE expression, meanwhile improving the contents of excitatory and inhibitory neurotransmitters, and ultimately leading to the impairment of the mouse brain. Further investigation is needed to elucidate the underlying mechanisms more definitively.

\section{Acknowledgments}

This work was generously supported by the College of Public Health, Shandong University. We are grateful to Professor Liu Ping, our supervisor, for her support and guidance. 


\section{REFERENCES}

1) Food and Drug Administration, HHS. 2003. Food labeling: trans fatty acids in nutrition labeling, nutrient content claims, and health claims. Final rule. Fed Regist 68 : 41433-41506.

2) Sun Q, Ma J, Campos H, Hankinson SE, Manson JE, Stampfer MJ, Rexrode KM, Willett WC, Hu FB. 2007. A prospective study of trans fatty acids in erythrocytes and risk of coronary heart disease. Circulation 115: 1858-1865.

3) Wang Q, Imamura F, Ma W, Wang M, Lemaitre RN, King IB, Song X, Biggs ML, Delaney JA, Mukamal KJ. 2015. Circulating and dietary trans fatty acids and incident type 2 diabetes in older adults: The Cardiovascular Health Study. Diabetes Care 38: 1099-1107.

4) Dalainas I, Ioannou HP. 2008. The role of trans fatty acids in atherosclerosis, cardiovascular disease and infant development. Int Angiol 27: 146-156.

5) Hung WL, Hsu BY, Tung YC, Ho CT, Hwang LS. 2016. Inhibitory effects of antioxidant vitamins against thiyl radical-induced trans fatty acid formation in PC-12 cells. J Funct Foods 21: 212-222.

6) Laake I, Carlsen MH, Pedersen JI, Weiderpass E, Selmer R, Kirkhus B, Thune I, Veierød MB. 2013. Intake of trans fatty acids from partially hydrogenated vegetable and fish oils and ruminant fat in relation to cancer risk. Int J Cancer 132: 1389-1403.

7) Grimm MO, Rothhaar TL, Grösgen S, Burg VK, Hundsdörfer B, Haupenthal VJ, Friess P, Kins S, Grimm HS, Hartmann T. 2012. Trans fatty acids enhance amyloidogenic processing of the Alzheimer amyloid precursor protein (APP). J Nutr Biochem 23: 1214-1223.

8) Hua-Hong YU, Liang ZS, Xiang-Mei LI, Qiu B, Liu R, Jing LI, Deng ZY. 2011. Effects of trans-fatty acids on damage of human umbilical vein endothelial cells. Food Sci 32: 244-247.

9) Pimentel GD, Lira FS, Rosa JC, Oliveira JL, LosinskasHachul AC, Souza GIH, Carmo MDGTD, Santos RVT, Mello MTD, Tufik S. 2012. Intake of trans fatty acids during gestation and lactation leads to hypothalamic inflammation via TLR4/NF $\kappa$ Bp65 signaling in adult offspring. J Nutr Biochem 23: 265-271.

10) Teixeira AM, Pase CS, Boufleur N, Roversi K, Barcelos RC, Benvegnú DM, Segat HJ, Dias VT, Reckziegel P, Trevizol F. 2011. Exercise affects memory acquisition, anxiety-like symptoms and activity of membrane-bound enzyme in brain of rats fed with different dietary fats: impairments of trans fat. Neuroscience 195: 80-88.

11) Phivilay A, Julien C, Tremblay C, Berthiaume L, Julien P, Giguère Y, Calon F. 2009. High dietary consumption of trans fatty acids decreases brain docosahexaenoic acid but does not alter amyloid-beta and tau pathologies in the 3xTg-AD model of Alzheimer's disease. Neuroscience 159: $296-307$.

12) Golomb BA, Bui AK. 2015. A fat to forget: Trans fat consumption and memory. PLoS One 10: e0128129.

13) Monguchi $T$, Ishida $T$, Nakajima $H$, Hasokawa $M$, Kondo K, Yasuda T, Hirata K. 2013. Trans fatty acids induce systemic inflammation and atherosclerosis through tolllike receptor-mediated pathway in ldl receptor knockout mice. Circulation 128: A14652.

14) Liu P, Wei Z, Tong Y. 2009. The research of NO and NOS' effects on learning memory and neurological toxicity. $J$ Liaoning Med Univ 30: 369-375.

15) Sharma HS. 2006. Hyperthermia influences excitatory and inhibitory amino acid neurotransmitters in the central nervous system. An experimental study in the rat using behavioural, biochemical, pharmacological, and morphological approaches. J Neural Transm 113: 497-519.

16) Lau A, Tymianski M. 2010. Glutamate receptors, neurotoxicity and neurodegeneration. Pflugers Arch 460: 525-542.

17) Xu G, Broadbelt KG, Haynes RL, Folkerth RD, Borenstein NS, Belliveau RA, Trachtenberg FL, Volpe JJ, Kinney HC. 2011. Late development of the GABAergic system in the human cerebral cortex and white matter. J Neuropathol Exp Neurol 70: 841-858. 